\title{
Micro- nano vehicles: Self-propelling approach toward the Future
}

\author{
Rabiee N* \\ Department of Chemistry, Shahid Beheshti University, Tehran, Iran
}

\begin{abstract}
Micro/nanomotors (MNM) described as tiny materials that can be able to producing autonomous movement in body fluids at minor range, ordinarily inside micro/ nano system. This developing subject is usually constantly bringing in innovative categories from distinct specialties, and shifting ahead with swift rate. Currently, scientists have got engineered and manufactured unique kinds of MNM, that are extra bio-friendly, effective and flexible in designs and components which can be able of moving forward in fluidic conditions. At the same time, numerous proof-of-concept manifestations on feasible functions of these MNM had been published which includes cargo delivery, chemical substance/biological sensing, cellular and tissues penetration, cellular delivery systems, wound healing and environmental remediation's. Entirely, researchers have got suggested and produced the initial attempt to employ these energetic carriers in the subject of nanomedicine.
\end{abstract}

The quick advancement of energetic, sensible carriers, including MNM, toward biomedical functions have been a study subject of substantial curiosity in fundamental technology, medication and actually in the general public opinion that we consider their precise apply cannot be very much in long term.

\section{Concepts and Opportunities}

Presently, nanoparticles-based medication delivery depends on the unaggressive transportation of the medication through the various moves experienced once nanoparticles are launched into living systems. Energetic transportation by using self-propelled nanoparticles (called nanomotors or nanorobots) is designed at improving the diffusion of the medication to particular places on demand. Preferably, they will conquer the runs or inside the spots wherever the diffusion is certainly extremely limit or the medicines cannot also penetrate, for example in which viscosity is certainly extremely substantial. Acquiring benefit of the managed locomotion of MNM can be able of medication launching and releasing, preferably these 'smart' MNM are anticipated to understand direct-targeted medication delivery in an energetic way. Therefore, the field of biomedicine can be evidently a perfect situation wherever self-propelled MNM can improve a big quantity of functions in the close to upcoming. Some proof-of-concept studies exhibited the delivery of anticancer medications by using self-propelled MNM, and actually additional medication liberating after achieving the focus on cellular material in vitro. Though a number of magazines on this subject possess been reported, not far off spaces among these demonstrations and practical biomedical make use of are rather apparent. In purchase to accomplish this kind of progressed energetic medication delivery automobile, there are many requirements have got to become convinced $[1,2,3,4]$.

The components of that the MNM can be produced by biocompatible materials. The energy featuring generating pressure for the propulsion should end up being obtainable in the liquid by itself, and preferably not really further externally (at least not really harmful to the organism). After that, the carriers need exact control on the directionality of their motion to reach the preferred focus on, which could become provided by exterior stimuli (magnet, electrical, ultrasound areas) or realizing in situ gradients $\mathrm{pH}$, heat, chemical substance structure). MNM should possess great cargo launching features in terms of weight transferred and as well on the kind of medicines packed. While, flexible strategies of medication delivery should end up being regarded as on demand. In this commentary, we will primarily focus on the biocompatibility concern once using MNM as biomedical carriers [5,6].

\section{Challenges and future prospective}

Even though there have got been substantial attempts on this study subject with critical improvement in latest years, it is definitely still a tremendous problem to fabricate a self-propelled strategy, which can accomplish all the criteria of reasonable biomedical process in vitro and in vivo. According to the current improvement of biomedical functions of MNM, the majority of the studies had been generally proof-of-concept manifestations, including striving at active-targeted medication delivery and transient or out of control energetic movement in natural environment.

Concerning chemically nature MNM, the majority of appealing strategy can be enzyme-powered MNM, seeing that it could be able to fabricate biocompatible motor body and make use of physiologically offered energy sources to power the engines. Additionally, common circular Janus engines in which case digestive enzymes had been asymmetrically immobilized on one part of the exterior surface area of a particle or nanorods to offer the generating push, experts also limited digestive enzymes in supramolecular stomatocyte and accomplished self-propulsion. The movement system of these enzyme-powered engines can be still conspiracy, and will need additional understanding for its advancement toward biomedical make use of. Particularly, the many encouraging strategy able of long-range self-propulsion is certainly generally approved to become run by phoretic system, which

${ }^{*}$ Correspondence to: Navid Rabiee, Invited Scientist, Department of Chemistry, Shahid Beheshti University, Tehran, Iran, E-mail: nrabiee94@gmail.com

Received: July 12, 2018; Accepted: July 25, 2018; Published: July 27, 2018 
demonstrated sensitivity toward ionic power. The stop of movement with electrolytes at millimolar range makes these enzyme engines ineffective when taking into consideration hundreds of millimolar electrolytes focus in natural liquid. In mixture with commendable metallic, to illustrate, $\mathrm{Pt}$ and $\mathrm{Au}$, the photocatalytic reactions to power engines may move in natural liquid under light direct exposure, but its energetic movement also suffers from ionic impact. Even though we attained the advancement of engines that can end up being powered by non-toxic gas, for factors still not really completely comprehended, their movement system is usually not really feasible within the natural environment. Consequently, long term analysis will need to bring out in-depth research on the fundamental understanding on the phoretic movement of these bio-friendly fuel-driven engines, in the wish of developing chemically driven engines able of conquering ionic impact. After that, that will become a stepping stone against any biomedical study including targeted transport of anticancer medications toward growth site in vivo.

For exterior field-powered MNM depending on gasoline free steam system, their movement will not really stop with the ionic impact. Except comparable difficulties provided by viscosity and difficulty of the actual natural liquid that hinders chemically run MNM, another very easily not far off problems for exterior field-powered MNM is definitely the problem of exactly applying exterior field to offer constant propulsion power in vivo. For example, cells penetration and light attenuation may impact the overall performance of light-powered engines for in vivo applications. And as well, in vivo light intro and constant light monitoring on the shifting engines will provide additional problems as well. The exterior field powered MNM generally needs a difficult and accurate area of the field used on the placement of the engines, which will certainly provide more hurdles toward practical utilization of these engines in individual body $[7,8,9,10,11,12]$.

As a result, rather than straight going for proof-of-concept presentations of potential biomedical functions of a recently created $\mathrm{MNM}$, additional critical research are extremely required to explore the biomedical potential of these MNM from the underlying. Concerning this element, it can be required to bring out inspections on the stationary connection between genuine bio-fluid with probable biomedical MNM. It can be also suggested to explore the movement behaviour of MNM in extremely viscous press, simulating particular bio-environment like the bones, wherever viscosity is certainly high and diffusion of medication is usually a problem. The energetic movement of MNM might help to deliver medicines into these areas wherever the diffusion of medications is definitely limited. Another feasible path may be a different perspective from outside of current goals for biomedical usage of MNM, in addition to the majority of generally discovered purpose of active-targeted medication delivery. And interdisciplinary relationships with additional areas are extremely suggested, specifically with experts or clinicians, which could be capable to encourage new biomedical applications of MNM, by producing great utilization of its tiny sizes and energetic movement ability $[13,14,15-18]$.

\section{References}

1. Chen C, Chang X, Teymourian H, Ramírez-Herrera DE, et al. (2018) Bioinspired chemical communication between synthetic nanomotors. Angew Chem Int Ed Engl 57: 241-245. [Crossref]

2. Díez P, Esteban-Fernández de Ávila B, Ramírez-Herrera DE, Villalonga R, Wang J (2017) Biomedical nanomotors: efficient glucose-mediated insulin release. Nanoscale 9: 14307-14311. [Crossref]

3. Liang H, Zhou B, Li J, et al. (2018) Engineering multifunctional coatings on nanoparticles based on oxidative coupling assembly of polyphenols for stimuliresponsive drug delivery. J Agric Food Chem 66: 6897-6905. [Crossref]

4. Sokolov IL, Cherkasov VR, Tregubov AA, Buiucli SR, Nikitin MP (2017) Smart materials on the way to theranostic nanorobots: Molecular machines and nanomotors, advanced biosensors, and intelligent vehicles for drug delivery. Biochimica Biophysica Acta 1861: 1530-1544. [Crossref]

5. Tu Y, Peng F, Wilson DA (2017) Motion manipulation of micro- and nanomotors. $A d v$ Mater 29. [Crossref]

6. Tu Y, Peng F, André AA, Men Y, Srinivas M, et al. (2017) Biodegradable hybrid stomatocyte nanomotors for drug delivery. ACS Nano 11: 1957-1963. [Crossref]

7. Angell C, Kai M, Xie S, Dong X, Chen Y (2018) Bioderived DNA nanomachines for potential uses in biosensing, diagnostics, and therapeutic applications. Advanced healthcare materials 7: 1701189. [Crossref]

8. Hritwick B, Shen S, Ren H (2018) Magnetically actuated minimally invasive microbots for biomedical applications. Electromagnetic Actuation and Sensing in Medical Robotics 11-41.

9. Choi H, Lee GH, Kim KS, Hahn SK (2018) Light-Guided nanomotor systems for autonomous photothermal cancer therapy. ACS applied materials \& interfaces 10: 2338-2346. [Crossref]

10. Berta Esteban-Fernández de Ávila, Angsantikul P, Li J, Gao W, Zhang L (2018) Micromotors go in vivo: from test tubes to live animals. Advanced Functional Materials 28: 1705640.

11. Jurado-Sánchez B and Wang J (2018) Micromotors for environmental applications: a review. Environmental Science: Nano

12. Konstantin GS, Vladimir RC, Irina LN, Andrey VB, Maxim PN (2018) Smart multifunctional nanoagents for in situ monitoring of small molecules with a switchable affinity towards biomedical targets. Applied Nanoscience 8: 195-203.

13. Baeza A, Vallet-Regí M (2018) Nanomotors for Nucleic Acid, Proteins, Pollutants and Cells Detection. International Journal of Molecular Sciences 19: 1579. [Crossref]

14. Ibrahim Y, Golestanian R, Liverpool TB (2017) Multiple phoretic mechanisms in the self-propulsion of a Pt-insulator Janus swimmer. Journal of Fluid Mechanics 828: 318352

15. Moran JL, Posner JD (2017) Phoretic self-propulsion. Annual Review of Fluid Mechanics 49: 511-540.

16. Patiño T, Feiner-Gracia N, Arqué X, Miguel-López A, Jannasch A, et al. (2018) Influence of enzyme quantity and distribution on the self-propulsion of non-janus urease-powered micromotors. J Am Chem Soc 140: 7896-7903. [Crossref]

17. Ibon S (2018) Nanoscale active matter matters: Challenges and opportunities for selfpropelled nanomotors. Nano Today 19: 11-15.

18. Santiago I, Jiang L, John F, and Turberfield AJ (2018) Self-propulsion of catalytic nanomotors synthesised by seeded growth of asymmetric platinum-gold nanoparticles. Chemical Communications 54: 1901-1904.

Copyright: (C2018 Rabiee N. This is an open-access article distributed under the terms of the Creative Commons Attribution License, which permits unrestricted use, distribution, and reproduction in any medium, provided the original author and source are credited. 